\title{
West Haven Hepatic Encephalopathy Grade 2
}

National Cancer Institute

\section{Source}

National Cancer Institute. West Haven Hepatic Encephalopathy Grade 2. NCI Thesaurus.

Code C117826.

Moderate - symptoms or pathological characteristics include lethargy or apathy, disorientation, inappropriate behavior, slurred speech, obvious asterixis, drowsiness, lethargy, gross deficits in ability to perform mental tasks, obvious personality changes, inappropriate behavior, and intermittent disorientation, unsurely regarding time. (Adapted from: Hepatic Encephalopathy. David C Wolf.

http://emedicine.medscape.com/article/186101-overview.) 